\title{
ON NUMERICAL-ANALYTIC TECHNIQUES FOR BOUNDARY VALUE PROBLEMS
}

\author{
András RONTÓ*, Miklós RONTÓ**, Nataliya SHCHOBAK*** \\ *Institute of Mathematics, Academy of Sciences of Czech Republic, Žižkova 22, 61662 Brno, Czech Republic, \\ e-mail: ronto@math.cas.cz \\ **Department of Analysis, University of Miskolc, 3515 Miskolc-Egyetemváros, Hungary, e-mail: matronto@ gold.uni-miskolc.hu \\ *** Uzhgorod National University, 46 Pidhirna St., 88000 Uzhgorod, Ukraine, e-mail: natasha.shchobak@ gmail.com
}

\begin{abstract}
We discuss several facts related to numerical-analytic methods for boundary value problems for first order ordinary and functional differential equations. A numerical-analytic scheme of investigation of a two-point boundary value problem for functional differential equations is stated.
\end{abstract}

Keywords: numerical-analytic method, periodic successive approximations, Lyapunov-Schmidt method

\section{INTRODUCTION}

It may be well agreed upon the philosophical thesis that the two basic questions for any boundary value problem are how to:

(E) Prove the existence of a solution in a given set.

(A) Choose a suitable approximation scheme that can be used for its practical finding.

As a rule, these two points are treated by methods that essentially differ from one another both in their nature and, what is most unhappy, in the prerequisites needed to guarantee their applicability. Indeed, the powerful armoury of topological methods, which can be regarded as a source of the majority of existence theorems and allows one to treat very rich classes of equations in a truly sophisticated manner, is firmly focused on answering question (E), providing only the very fact of the existence of a solution as the ultimate result, with some or another additional information on its spatial localisation at most, and usually no hints for its efficient construction. In other words, techniques based on a priori estimates and continuation, as a rule, allow one to study question (E) only.

On the other hand, with very few exceptions, all the numerical methods providing one tools for addressing question (A), can only be applied when the solvability of the problem under consideration is known, i. e., one should assume that $(\mathrm{E})$ has already been solved.

One thus has to deal with two or more kinds of techniques to solve both (E) and (A). This is the case, for example, when Galerkin-type methods are used to construct a solution the existence of which has been proved by using topological methods [8]. The choice of a suitable combination of methods, however, may be quite problematic because the applicability conditions are usually different. This is the case, for example, when, for a problem with discontinuities, some conditions providing a priori estimates turn out to be sufficient for solving (E), whereas for the convergence of a discretisation scheme leading one to the resolution of (A), some smoothness restrictions are needed. Such a situation is rather generic.

In order to overcome this complication, one may choose either to renounce the mathematical rigorousness to some extent by relying on one's intuition and treating (A) without a complete substantiation of the discretisation method in use, or to try to apply an approach that would, under suitable assumptions, help in solving both (E) and (A) with sufficient strictness.

The latter choice is a matter of general expediency and personal preference. In particular, when the non-linear terms exhibit some kind of monotone behaviour, it makes sense to try the two-sided approximation methods (or, in an alternative terminology, monotone-iterative techniques [6, 9]), having their origin in the method introduced by Chaplygin in 1919 (see [2, 10,13]). In case of success, one proves the solvability of the problem and constructs two sequences that approximate the solutions from below and from above respectively with increasing accuracy. However, the key assumption allowing one to apply such techniques requires the existence of the initial couple of approximations which, in the theory of differential equations, are usually called lower and upper functions (see, e. g., [15]). The latter non-trivial assumption, as a rule, is not easy to verify, and even more, the question on the construction of such a pair of elements may turn out to be comparable in complexity with the original problem. The interested reader may refer to the book [14] for a clear exposition of this matter in a general setting involving heterotone operators (now frequently called mixed monotone, apparently due to [4]).

The aim of this note is to outline the advantages that the so-called numerical-analytic methods may have when one is interested in treating (E) and (A) simultaneously. The idea dates back to the works of Lyapunov and Schmidt [11,37] and suggests one to decompose the space in such a way that the given operator equation, under appropriate assumptions, is reduced to a system of finitely many numerical equations usually referred to as determining equations (see [3, 7, 39, 40] for a detailed exposition).

We are interested in the efficient realisation of this idea developed by Samoilenko [33, 34], motivated by [1,5], and, in the periodic case, known under the name method of periodic successive approximations [17,35]. We refer the reader to [17, 24, 35, 36] for more details. Many comments and references on this topic can also be found in the survey [25-31]. Among recent papers devoted to the subject discussed, we mention [18,-21, 23]. 
Here, we breafly describe an approach of this kind adopted to two-point boundary value problems for systems of functional differential equations.

Consider the system of functional differential systems

$x^{\prime}(t)=(f x)(t), \quad t \in[a, b]$,

determined by a non-linear operator $f: C\left([a, b], \mathbb{R}^{n}\right) \rightarrow$ $L_{1}\left([a, b], \mathbb{R}^{n}\right)$. Equation $(1)$ is studied under the two-point linear boundary conditions of a non-separated type

$A x(a)+B x(b)=d$,

where $d \in \mathbb{R}^{n}$ and $B$ is a non-singular matrix. Without loss of generality, one may restrict oneself to the boundary condition of the particular form

$A x(a)+x(b)=0$.

\section{SUCCESSIVE APPROXIMATIONS}

We look for a solution of problem (1), (3) among functions having initial value in the cone segment $\left\langle z_{0}, z_{1}\right\rangle$ of the form

$$
\left\langle z_{0}, z_{1}\right\rangle:=\left\{z \in \mathbb{R}^{n} \mid z_{0} \leq z \leq z_{1}\right\},
$$

where $z_{0}$ and $z_{1}$ are certain fixed vectors. Geometrically, this means that we fix a strip-like region where $x(a)$ for a potential solution $x(\cdot)$ may vary. Here and below, the inequalities for vectors and matrices are understood in the componentwise sense.

Definition 2.1. A mapping $f: C\left([a, b], \mathbb{R}^{n}\right) \rightarrow L_{1}\left([a, b], \mathbb{R}^{n}\right)$ is said to satisfy the Lipschitz condition on a set $\mathscr{B} \subset$ $C\left([a, b], \mathbb{R}^{n}\right)$ if there exists a positive linear operator $l$ : $C\left([a, b], \mathbb{R}^{n}\right) \rightarrow L_{1}\left([a, b], \mathbb{R}^{n}\right)$ such that

$$
|(f u)(t)-(f v)(t)| \leq(l|u-v|)(t), \quad t \in[a, b],
$$

for all $u$ and $v$ from $\mathscr{B}$.

The positivity of $l$ in the last definition is understood in the following sense.

\section{Definition 2.2. An operator}

$l: C\left([a, b], \mathbb{R}^{n}\right) \rightarrow L_{1}\left([a, b], \mathbb{R}^{n}\right)$

is said to be positive if $(l u)(t) \geq 0$ for a.e. $t \in[a, b]$ whenever $u(t) \geq 0$ for all $t \in[a, b]$.

The Lipschitz condition (5) will be assumed in tube-like regions. More precisely, given any vectors $y_{0}$ and $y_{1}$ from $\mathbb{R}^{n}$, we define the set $\mathscr{B}\left(y_{0}, y_{1}\right)$ by putting

$$
\begin{array}{r}
\mathscr{B}\left(y_{0}, y_{1}\right):=\left\{x \in C\left([a, b], \mathbb{R}^{n}\right): y_{0} \leq x(t) \leq y_{1}\right. \\
\text { for all } t \in[a, b]\} .
\end{array}
$$

In the sequel, we restrict our consideration to the case where the positive linear operator $l: C\left([a, b], \mathbb{R}^{n}\right) \rightarrow$ $L_{1}\left([a, b], \mathbb{R}^{n}\right)$ appearing in the Lipschitz condition (5) possesses the property

$\underset{t \in[a, b]}{\operatorname{ess} \sup } \frac{\left(l_{i j} \sigma\right)(t)}{\sigma(t)}<+\infty$ for all $i, j=1,2, \ldots, n$, where

$\sigma(t):=(t-a)(b-t), \quad t \in[a, b]$,

and the operators $l_{i j}: C([a, b], \mathbb{R}) \rightarrow L_{1}([a, b], \mathbb{R}), i, j=$ $1,2, \ldots, n$, are the components of $l$ defined according the formula

$\left(l_{i k} u\right)(t):=l_{i}\left(u e_{k}\right), \quad t \in[a, b]$,

for all $i, j=1,2, \ldots, n$ and $u \in C([a, b], \mathbb{R})$.

It is clear that, under assumption (7), the components

$v_{i j}:=\operatorname{esssup}_{t \in[a, b]} \frac{1}{\sigma(t)}\left(l_{i j} \sigma\right)(t)$

of the square matrix

$V:=\left(v_{i j}\right)_{i, j=1}^{n}$

are finite for any $i, j=1,2, \ldots, n$. Since $\sigma$ is a non-negative function and the operator $l$ is positive, it is clear that all the elements of $V$ are non-negative. Inequality (7), in fact, is a growth restriction for the components of $l$.

Our numerical-analytic study of solutions of the boundary value problem (1), 3 is based upon the use of the function sequence determined by the recurrence relation

$x_{m+1}(\cdot, z):=P f x_{m}(\cdot, z)+\varphi_{z}, \quad m=0,1,2, \ldots$,

with $x_{0}(\cdot, z):=\varphi_{z}$, where

$\varphi_{z}(t):=z-\frac{t-a}{b-a}\left(A+\mathbf{1}_{n}\right) z, \quad t \in[a, b]$,

for any $z \in\left\langle z_{0}, z_{1}\right\rangle$ and

$(P y)(t):=\int_{a}^{t} y(s) d s-\frac{t-a}{b-a} \int_{a}^{b} y(s) d s$

for all $y \in L_{1}\left([a, b], \mathbb{R}^{n}\right)$ and $t \in[a, b]$. The vector $z$ in $[12)$ is considered as an unknown parameter varying between $z_{0}$ and $z_{1}$. The projector $P$ arises here in a natural way (see, e. g., [17,35] and [32, p. 88]).

It is easy to verify that, for every $m=0,1,2, \ldots$ function (12) satisfies the boundary condition (3) for an arbitrary value of $z$. In what follows, this important observation allows one to "forget" about the boundary condition because all the functions that can potentially be considered as approximations already satisfy it.

Let us introduce into consideration the $n \times n$ matrices $\bar{A}_{-}=\left(\bar{a}_{-; i, j}\right)_{i, j=1}^{n}$ and $\overline{\bar{A}}_{-}=\left(\overline{\bar{a}}_{-; i, j}\right)_{i, j=1}^{n}$ with the elements defined by the equalities

$\bar{a}_{-; i, j}:= \begin{cases}0 & \text { if } i \neq j \\ \min \left\{1,\left[a_{i i}\right]_{-}\right\} & \text {if } i=j\end{cases}$

and

$\overline{\bar{a}}_{-; i, j}:= \begin{cases}{\left[a_{i j}\right]_{-}} & \text {if } i \neq j, \\ \max \left\{1,\left[a_{i i}\right]_{-}\right\} & \text {if } i=j .\end{cases}$

Finally, we put

$\omega(z):=\underset{t \in[a, b]}{\operatorname{ess} \sup }\left(f \varphi_{z}\right)(t)-\underset{t \in[a, b]}{\operatorname{essinf}}\left(f \varphi_{z}\right)(t)$

for all $z \in\left\langle z_{0}, z_{1}\right\rangle$, where $\varphi_{z}$ is the function defined by (13). 


\section{CONVERGENCE}

Assumption (7) allows one to prove the following statement.

Theorem 3.1. Assume that $f$ satisfies the Lipschitz condition (5) on the set $\mathscr{B}\left(-\rho_{*}+\bar{A}_{-} z_{0}-A_{+} z_{1}, \overline{\bar{A}}_{-} z_{1}+\rho_{*}\right)$, where

$\rho_{*}:=\frac{3}{4}\left(\frac{3}{b-a} \mathbf{1}_{n}-V\right)^{-1} \sup _{\xi \in\left\langle z_{0}, z_{1}\right\rangle} \omega(\xi)$

and $l: C\left([a, b], \mathbb{R}^{n}\right) \rightarrow L_{1}\left([a, b], \mathbb{R}^{n}\right)$ is a certain positive linear operator with property (7). Furthermore, assume that the corresponding matrix $V=\left(v_{i j}\right)_{i, j=1}^{n}$ with elements 10 , satisfies the condition

$r(V)<\frac{3}{b-a}$.

\section{Then:}

1. For any fixed $z \in\left\langle z_{0}, z_{1}\right\rangle$, the sequence of functions (12) converges uniformly on $[a, b]$ to a function

$$
x_{\infty}(\cdot, z):=\lim _{m \rightarrow \infty} x_{m}(\cdot, z)
$$

possessing the properties

$$
\begin{aligned}
x_{\infty}(a, z) & =z, \\
A x_{\infty}(a, z)+x_{\infty}(b, z) & =0 .
\end{aligned}
$$

2. The formula

$$
\begin{aligned}
\left\langle z_{0}, z_{1}\right\rangle \ni z \longmapsto \Delta(z):= & \left(A+\mathbf{1}_{n}\right) z \\
& +\int_{a}^{b}\left(f x_{\infty}(\cdot, z)\right)(s) d s
\end{aligned}
$$

introduces a well defined single-valued function $\Delta$ : $\left\langle z_{0}, z_{1}\right\rangle \rightarrow \mathbb{R}^{n}$.

3. The limit function (20) for all fixed $z \in\left\langle z_{0}, z_{1}\right\rangle$ is a solution of the Cauchy problem

$x^{\prime}(t)=(f x)(t)-\Delta(z), \quad t \in[a, b]$,

$x(a)=z$,

where the vector function $\Delta:\left\langle z_{0}, z_{1}\right\rangle \rightarrow \mathbb{R}^{n}$ is given by 21.

4. For all fixed $z \in\left\langle z_{0}, z_{1}\right\rangle$ and $m \geq 1$, the estimate

$$
\max _{t \in[a, b]}\left|x_{\infty}(t, z)-x_{m}(t, z)\right| \leq \sigma(t) \frac{(b-a)^{m-1}}{3^{m}} V^{m} \tilde{V} \omega(z)
$$

holds, where

$$
\tilde{V}:=\left(\mathbf{1}_{n}-\frac{1}{3}(b-a) V\right)^{-1} .
$$

It is important to obverve that the Lipschitz condition (5) in Theorem 3.1 is assumed on the bounded set $\mathscr{B}\left(-\rho^{*}+\bar{A}_{-} z_{0}-A_{+} z_{1}, \bar{A}_{-} z_{1}+\rho^{*}\right)$ only and, in general, may not be satisfied globally. The convergence of the successive approximations is then guaranteed by the smallness condition (19) for the eigenvalues of the matrix $V$.

One can specify other convergence conditions which do not depend on property (7]) (see, e. g., [23]). Note, however, that assumption (7) is satisfied in many cases. For example, if the components of the Lipschitz operator $l$ in (5) have the form

$\left(l_{i k} u\right)(t):=p_{i k}(t) u\left(\tau_{i k}(t)\right), \quad t \in[a, b], i, k=1,2, \ldots, n$,

with $p_{i k}:[a, b] \rightarrow \mathbb{R}$ integrable and $\tau_{i k}:[a, b] \rightarrow[a, b]$ measurable, it follows immediately from the relation

$\frac{\left(l_{i j} \sigma\right)(t)}{\sigma(t)}=p_{i j}(t) \frac{\left(\tau_{i j}(t)-a\right)\left(\tau_{i j}(t)-b\right)}{(t-a)(t-b)}$

that (7) holds, in particular, if either the function

$[a, b] \ni t \longmapsto \frac{p_{i j}(t)}{(t-a)(t-b)}$

is essentially bounded or $p_{i j} \in L_{\infty}([a, b], \mathbb{R})$ and $\tau_{i j}(t) \leq t$, $i, j=1,2, \ldots, n$.

The following general statement on the solvability of the boundary value problem (1), (3) holds.

Theorem 3.2. Let the conditions of Theorem 3.1 be satisfied. Then the limit function $x_{\infty}(\cdot, z)$ of the recurrence sequence (12) is a solution of the boundary value problem (1), (3) if, and only if the value of the vector parameter $z \in\left\langle z_{0}, z_{1}\right\rangle$ satisfies the system of equations

$\Delta(z)=0$,

where $\Delta:\left\langle z_{0}, z_{1}\right\rangle \rightarrow \mathbb{R}^{n}$ is given by 21 .

Equations of type (26) are usually referred to as determining equations [3, 35] because they determine the actual values of the parameters $z \in\left\langle z_{0}, z_{1}\right\rangle$ involved in the iteration process (12). Likewise, $\Delta:\left\langle z_{0}, z_{1}\right\rangle \rightarrow \mathbb{R}^{n}$ given by (21) is often called a determining function for problem (1), (3).

Theorem 3.2 reduces the boundary value problem (1), (3) to the finite-dimensional system of equations (26).

\section{APPROXIMATE DETERMINING EQUATIONS}

The main difficulty related to the determining system (26) is that the explicit form of the vector field $\Delta$ is unknown. A constructive investigation of problem (1), (3) with the help of Theorem 3.2 is carried out by passing from the exact determining equation 26 to some its approximations. In practice, it is natural to fix some $m \geq 1$, introduce the $m$ th approximate determining function $\Delta_{m}:\left\langle z_{0}, z_{1}\right\rangle \rightarrow$ $\mathbb{R}^{n}$ by setting

$\Delta_{m}(z):=\left(A+\mathbf{1}_{n}\right) z+\int_{a}^{b}\left(f x_{m}(\cdot, z)\right)(s) d s$

for all $z \in\left\langle z_{0}, z_{1}\right\rangle$ and, instead of the inconvenient equation (26), consider the $m$ th approximate determining equation of the form

$\Delta_{m}(z)=0$. 
It should be noted that, in contrast to (26), the new equation (28) is constructed directly based on the function $x_{m}(\cdot, z)$ and does not involve any unknown terms. It turns out that, under suitable assumptions, the function

$X_{m}(t):=x_{m}(t, \tilde{z}), \quad t \in[a, b]$,

where $\tilde{z}$ is a root of the system of equations $(28)$, can be regarded as an $m$ th approximation to a solution of problem (1), (3).

We need a definition describing a kind of the strict inequality for vector functions.

Definition 4.1. Let $S \subset \mathbb{R}^{n}$ be an arbitrary non-empty set. For any pair of functions $g_{j}=\operatorname{col}\left(g_{j, 1}, \ldots, g_{j, n}\right), j=1,2$, we write

$g_{1} \triangleright_{S} g_{2}$

if and only if there exists a function $v: S \rightarrow\{1,2, \ldots, n\}$ such that the strict inequality

$g_{1, v(x)}(x)>g_{2, v(x)}(x)$

holds for all $x \in S$.

The following statement gives conditions sufficient for the solvability of the boundary value problem (1), 3) based on properties of a certain fixed member of the recurrence sequence (12).

Theorem 4.1. Let us suppose that, in addition to assumptions of Theorem 3.1. there exist a closed domain $\Omega \subset$ $\left\langle z_{0}, z_{1}\right\rangle$ and an integer $m \geq 1$ such that, on the boundary of $\Omega$, the approximate determining function $\Delta_{m}$ given by formula (27) satisfies the condition

$\left|\Delta_{m}\right| \triangleright_{\partial \Omega} \frac{1}{2} \frac{(b-a)^{m+2}}{3^{m+1}} V^{m+1} \tilde{V} \omega$,

where $\omega:\left\langle z_{0}, z_{1}\right\rangle \rightarrow \mathbb{R}^{n}$ is the function given by (17) and $\tilde{V}$ is the matrix (24).

Let, moreover,

$\operatorname{deg}\left(\Delta_{m}, \Omega, 0\right) \neq 0$

Then there exists a certain $z^{*} \in \Omega$ such that the function $x_{\infty}\left(\cdot, z^{*}\right)$ is a solution of the boundary value problem (1), (3).

It follows from the last theorem that, once the existence of a solution is proved on the $m$ th step of iteration after the verification of conditions (32) and (33), one is also able to construct its approximation according to formula $(29)$ by solving the system of $n$ numerical equations (28) the form of which is known explicitly. The corresponding error estimates are derived from the properties of the recurrence sequence (12)

\section{COMMENTS}

The following features of the scheme described above should be mentioned:
- The Lipschitz condition is assumed on a bounded set.

- There is no assumption on the existence and uniqueness of a solution of the Cauchy problem. Moreover, in the class of equations (1), there is no unique solvability of this problem in general (which property, in particular, affects shooting methods [38]). It is also essential that this is not a matter of sufficient smoothness of coefficients: for example, the initial value problem

$u(a)=0$

for the simplest functional differential equation

$u^{\prime}(t)=\frac{u(b)}{b-a}+q(t), \quad t \in[a, b]$,

where $-\infty<a<b<\infty$ and $q:[a, b] \rightarrow \mathbb{R}$ is such that $\int_{a}^{b} q(s) d s \neq 0$, has no solution.

Note that 34 is a linear equation with the constant coefficient $(b-a)^{-1}$, which, in addition, is small on large intervals.

- In contrast to Galerkin-type methods, there is no need to recalculate all the data when passing to the next step.

- The scheme works well on tiny intervals. For example, when looking for $2 \pi \omega^{-1}$-periodic solutions with, e.g., $\omega=10^{10}$, one does not have to discretise the equation with step less than $10^{-11}$.

- Can be adopted for equations with various kinds of argument deviations and more complicated boundary conditions (see, e. g., [16, 17, 22]).

- In contrast to monotone-iterative methods, there are no difficulties with the selection of the starting approximation (indeed, the function $\varphi_{z}$ in 12 is constructed directly according to 13 using the form of the boundary condition (3)).

- One can combine this approach with other techniques (e. g., polynomial interpolation [21 36]) that facilitate the realisation of its analytic part.

- The approximate solution, constructed explicitly in a finite number of steps, helps in proving the existence of an exact one.

\section{ACKNOWLEDGEMENT}

This research supported in part by RVO: 67985840 (A. Rontó) was carried out as part of the TAMOP-4.2.1.B10/2/KONV-2010-0001 project with support by the European Union, co-financed by the European Social Fund (M. Rontó).

\section{REFERENCES}

[1] CESARI, L.: Asymptotic Behavior and Stability Problems in Ordinary Differential Equations. 2nd edition. Ergebnisse der Mathematik und ihrer Grenzgebiete, N. F., Bd. 16. Academic Press Inc., New York, 1963. 
[2] CHAPlygin, S. A.: Collected Works [in Russian], Vol. I, Moscow, 1948.

[3] GAINES, R. - MAWHIN, J.: Coincidence Degree, and Nonlinear Differential Equations. Lecture Notes in Mathematics 568, Springer-Verlag, BerlinHeidelberg-New York, 1977.

[4] GUO, DA JUN - LAKSHMIKANTHAM, V.: Coupled fixed points of nonlinear operators with applications, Nonlinear Anal. 11, No. 5 (1987) 623-632.

[5] HALE, J. K.: Oscillations in Nonlinear Systems, McGraw-Hill Book Co., Inc., New York-TorontoLondon, 1963.

[6] HEIKKILÄ, S. - LAKSHMIKANTHAM, V.: Monotone Iterative Techniques for Discontinuous Nonlinear Differential Equations. Monographs and Textbooks in Pure and Applied Mathematics, 181. Marcel Dekker, Inc., New York, 1994, ISBN 0-8247-9224-6.

[7] KNOBLOCH, H. W.: Remarks on a paper of L. Cesari on functional analysis and nonlinear differential equations, Michigan Math. J. 16, No. 4, 417-430 (1963).

[8] KRASNOSELSKII, M. A. - VAINIKKO, G. M. ZABREIKO, P. P. - RUTITSKII, YA. B. - STETSENKO, V. Y.: Approximate Solution of Operator Equations. Translated from the Russian by D. Louvish. Wolters-Noordhoff Publishing, Groningen, 1972.

[9] LADDE, G. S. - LAKSHMIKANTHAM, V. - VATSALA, A. S.: Monotone iterative techniques for nonlinear differential equations. Monographs, Advanced Texts and Surveys in Pure and Applied Mathematics, 27. Pitman (Advanced Publishing Program), Boston, MA; distributed by John Wiley \& Sons, Inc., New York, 1985, ISBN 0-273-08707-X.

[10] LUSIN, N. N.: On S. A. Chaplygin's Method of Approximate Integration [in Russian], State Central Aerodynamics Inst., No. 141, 1932.

[11] LYAPUNOV, A. M.: Sur les figures d'équilibre peu différentes des ellipsö̈des d'une masse liquide homogène dotée d'un mouvement de rotation, Zap. Akad. Nauk. St. Petersbourg, 1906, 1-225.

[12] MAWHIN, J.: Topological Degree Methods in Nonlinear Boundary Value Problems, volume 40 of CBMS Regional Conference Series in Mathematics. American Mathematical Society, Providence, R.I., 1979. ISBN 0-8218-1690-*. Expository lectures from the CBMS Regional Conference held at Harvey Mudd College, Claremont, Calif., June 9-15, 1977.

[13] MIKHLIN, S. G. - SMOLITSKIY, K. L.: Approximate Methods for Solution of Differential and Integral Equations. Translated from the Russian by Scripta Technica, Inc., Modern Analytic and Computational Methods in Science and Mathematics, Vol. 5, American Elsevier Publishing Co., Inc., New York, 1967.

[14] OPOITSEV, V. I.: Nonlinear Systemostatics [in Russian], Library of Mathematical Economics, 31. "Nauka", Moscow, 1986.
[15] RACHŮNKOVÁ, I. - STANĚK, S. - TVRDÝ, M.: Solvability of Nonlinear Singular Problems for Ordinary Differential Equations, Hindawi Publishing Corp., Cairo, 2009, ISBN 9-7745-4040-9.

[16] RONTÓ, A. - RONTÓ, M.: A note on the numericalanalytic method for nonlinear two-point boundaryvalue problems, Nonlinear Oscil. 4, No. 1 (2001) 112 128.

[17] RONTÓ, A. - RONTÓ, M.: Successive Approximation Techniques in Non-Linear Boundary Value Problems for Ordinary Differential Equations. In Handbook of differential equations: ordinary differential equations. Vol. IV, Handb. Differ. Equ., pages 441592, Elsevier/North-Holland, Amsterdam, 2008.

[18] RONTÓ, A. - RONTÓ, M.: On nonseparated three-point boundary value problems for linear functional differential equations, Abstr. Appl. Anal. 2011, Art. ID 326052 (2011), 1-22. ISSN 1085-3375, DOI 10.1155/2011/326052, http://dx.doi.org/10. 1155/2011/326052.

[19] RONTÓ, A. - RONTÓ, M.: Existence results for three-point boundary value problems for systems of linear functional differential equations, Carpathian J. Math. 28, No. 1 (2012) 163-182.

[20] RONTÓ, A. - RONTÓ, M.: On constructive investigation of a class of non-linear boundary value problems for functional differential equations, Carpathian J. Math., to appear.

[21] RONTÓ, A. - RONTÓ, M. - HOLUBOVÁ, G. - NEČESAL, P.: Numerical-analytic technique for investigation of solutions of some nonlinear equations with Dirichlet conditions, Boundary Value Problems, 2011:58 (2011), 1-20, ISSN 1687-2770, DOI 10.1186/1687-2770-2011-58, http://dx.doi. org/10.1186/1687-2770-2011-58

[22] RONTÓ, A. - RONTÓ, M. - SHCHOBAK, N.: On the parametrization of three-point nonlinear boundary-value problems, Nonlinear Oscillations 7, No. 3 (2004) 384-402.

[23] RONTÓ, A. - RONTÓ, M. - SHCHOBAK, N.: On finding solutions of two-point boundary value problems for a class of non-linear functional differential systems, Electron. J. Qual. Theory Differ. Equ., No. 13 (2012), 1-17.

[24] RONTÓ, M. - SAMOILENKO, A.: NumericalAnalytic Methods in the Theory of Boundary-Value Problems. World Scientific Publishing Co. Inc., 2000, ISBN 981-02-3676-X. [With a preface by Yu. A. Mitropolsky and an appendix by the authors and S. I. Trofimchuk.]

[25] RONTO, N. I. - SAMOILENKO, A. M. - TROFIMCHUK, S. I.: The theory of the numerical-analytic method: achievements and new directions of development. I, Ukrainian Math. J. 50, No. 1 (1998) 116-135.

[26] RONTO, N. I. - SAMOILENKO, A. M. - TROFIMCHUK, S. I.: The theory of the numerical-analytic 
method: achievements and new directions of development. II, Ukrainian Math. J. 50, No. 2 (1998) 255277.

[27] RONTO, N. I. - SAMOILENKO, A. M. - TROFIMCHUK, S. I.: The theory of the numerical-analytic method: achievements and new directions of development. III, Ukrainian Math. J. 50, No. 7 (1998) 10911114.

[28] RONTO, N. I. - SAMOILENKO, A. M. - TROFIMCHUK, S. I.: The theory of the numerical-analytic method: achievements and new directions of development. IV, Ukrainian Math. J. 50, No. 12 (1998) 18881907.

[29] RONTO, N. I. - SAMOILENKO, A. M. - TROFIMCHUK, S. I.: The theory of the numerical-analytic method: achievements and new directions of development. V, Ukrainian Math. J. 51, No. 5 (1999) 735 747.

[30] RONTO, N. I. - SAMOILENKO, A. M. - TROFIMCHUK, S. I.: The theory of the numerical-analytic method: achievements and new directions of development. VI, Ukrainian Math. J. 51, No. 7 (1999) 1079_ 1094.

[31] RONTO, N. I. - SAMOILENKO, A. M. - TROFIMCHUK, S. I.: The theory of the numerical-analytic method: achievements and new directions of development. VII, Ukrainian Math. J. 51, No. 9 (1999) 1399_ 1418.

[32] ROUCHE, N. - MAWHIN, J.: Ordinary Differential Equations. Stability and Periodic Solutions, volume 5 of Surveys and Reference Works in Mathematics. Pitman, Boston, 1980, ISBN 0-273-08419-4. [Translated from the French and with a preface by R. E. Gaines.]

[33] SAMOILENKO, A. M.: Numerical-analytic method for the investigation of periodic systems of ordinary differential equations. I, Ukr. Mat. Zh. 17, No. 4 (1965) 82-93.

[34] SAMOILENKO, A. M.: Numerical-analytic method for the investigation of periodic systems of ordinary differential equations. II, Ukr. Mat. Zh. 18, No. 2 (1966) 50-59.

[35] SAMOILENKO, A. M. - RONTO, N. I.: NumericalAnalytic Methods of Investigating Periodic Solutions. Mir, Moscow, 1979. [Translated from the Russian, with a foreword by Yu. A. Mitropolskii.]

[36] SAMOILENKO, A. M. - RONTO, N. I.: Numerical-Analytic Methods for Investigation of Solutions of Boundary-Value Problems [in Russian], Naukova Dumka, Kiev, 1985. [With an English summary, edited and with a preface by Yu. A. Mitropolskii.]

[37] SCHMIDT, E.: Zur Theorie des linearen und nichtlinearen Integralgleichungen. 3 Teil. Über die Auflösung der nichtlinearen Integralgleichungen und die Verzeigung ihrer Lözungen, Math. Ann. 65 (1908), 370-399.

[38] STOER, J. - BULIRSCH, R.: Introduction to Numerical Analysis. [Translated from the German by R. Bartels, W. Gautschi and C. Witzgall.] Springer-Verlag, New York-Heidelberg, 1980, ISBN 0-387-90420-4.

[39] VAINBERG, M. M. - TRENOGIN, V. A.: The methods of Lyapunov and Schmidt in the theory of nonlinear equations and their further development, Russian Math. Surveys 17, No. 1 (1962), 1-60, DOI: 10.1070/RM1962v017n02ABEH001127.

[40] ZABREIKO, P. P. - TIKHONOV, V. P.: Determining equations and the duality principle, Siberian Math. J. 24, No. 1 (1983), 65-72, DOI: 10.1007/BF00968797.

Received July 27, 2012, accepted September 26, 2012

\section{BIOGRAPHIES}

András Rontó was born on October 29, 1973. In 1995, graduated with distinction at the Faculty of Mechanics and Mathematics Informatics of the Faculty of Electrical Engineering and Informatics at University of Kiev. PhD (1998), DSc (2006). A researcher at the Institute of Mathematics of the Academy of Sciences of Czech Republic. His research interests cover the theory of functional differential equations, numerical-analytic methods, and boundary value problems.

Miklós Rontó was born on March 31, 1943. PhD (1971), DSc (1986). Since 1992, a Full Professor at the Department of Analysis of the University of Miskolc, Hungary. His research interests cover the theory of boundary value problems for ordinary differential equations. A co-author of several books on this topic.

Nataliya Shchobak was born on February 1, 1979. She graduated with distinction from the Uzhgorod National University in 2002. $\mathrm{PhD}$ (2007). She is an Associate Professor at the Uzhgorod National University. Her research interests lie in the field of constructive investigation of boundary value problems and numerical-analytic methods. 